Article

\title{
The Presence of a Powerful Retailer on Dynamic Collecting Closed-Loop Supply Chain From a Sustainable Innovation Perspective
}

\author{
Jackson Jinhong Mi ${ }^{1}$, Zongsheng Huang ${ }^{1,2} \mathbb{B}^{\mathbb{C}}$, Kai Wang ${ }^{3, *}$, Sang-Bing Tsai ${ }^{3,4, *(\mathbb{D})}$ \\ Guodong $\mathrm{Li}^{5, *}$ and Jiangtao Wang ${ }^{4}$ \\ 1 School of Economics and Management, Shanghai Maritime University, Shanghai 201306, China; \\ jhmi@shmtu.edu.cn (J.J.M.); zshuang@shmtu.edu.cn (Z.H.) \\ 2 School of Computer Science, Fudan University, Shanghai 200433, China \\ 3 College of Business Administration, Capital University of Economics and Business, Beijing 100070, China \\ 4 Zhongshan Institute, University of Electronic Science and Technology of China, Zhongshan 528402, China; \\ jiangtao-w@foxmail.com \\ 5 Economics and Management College, Civil Aviation University of China, Tianjin 300300, China \\ * Correspondence: wangkai@cueb.edu.cn (K.W.); sangbing@hotmail.com (S.-B.T.); gdli@cauc.edu.cn (G.L.)
}

Received: 4 May 2018; Accepted: 15 June 2018; Published: 21 June 2018

\begin{abstract}
In this study, the presence of a powerful retailer in the dynamic collecting closed-loop supply chain is considered. The supply chain consists of a manufacturer and a retailer who is responsible for the used-product collection. Specifically, we have considered three settings, that is, no channel leader, the manufacturer as the leader and the retailer as the leader. The steady equilibrium and the optimal control strategies are derived in different channel power structures utilizing the open-loop control strategy method. By the comparison of three models, we found that the system with manufacturer leading results in the lowest return rate in the three models. If the transfer price of the used-product is low, the presence of a power retailer would result in a higher return rate, which is beneficial for decreasing the market price for the consumers. When the transfer price is quite high, the scenario with no channel leader in the supply chain would result in a higher return rate. However, the numerical comparison results of the profit rate of the supply chain members indicate that both manufacturer and retailer prefer themselves to be the channel leader rather than the other channel member as the leader. This means that although the presence of a power retailer is beneficial for the collecting efficiency, it is not enough to increase the profit of the supply chain members. The manufacturer should transfer all unit cost savings to the retailer in the scenario of manufacturer leading and no one leading.
\end{abstract}

Keywords: closed-loop supply chain; differential game; open-loop control strategy; retailer collecting; channel power structure; sustainability; sustainable innovation

\section{Introduction}

Recently, the closed-loop supply chain has drawn increasing attention from academic, as it can significantly reduce the firm's production cost as well as reduce the pressure on the environment. Some large firms, such as Xerox, Kodak and HP, have already started to comprehensively employ the remanufacture strategy [1,2]. Collecting and the remanufacturing the end-of-life product is a good way to close the loop for the supply chain and it can also reduce waste emissions and resource consumption which meets the goal of sustainability (Mota et al.) [3]. Currently, the firms which employ a remanufacture strategy can be found in almost all kinds of industries, such as the computer industry, printer industry, ink-cartage industry, furniture industry, auto parts industry, medical 
equipment industry, etc. (Ferguson and Toktay, 2006) [4]. To employ a remanufacturing strategy, the most important procedure is to collect the used-products since used-products serve as materials for the remanufacturing.

However, used-product collecting is quite difficult because the used-products disperse over a large geography area. In reality, there are three different collecting modes across a supply chain, including, retailer collecting, manufacturer collecting and third party collecting. Figuring out the best collecting mode is a focus which has been intensely studied by the researchers during the last decade. Decentralization of the supply chain structure makes the program even harder to carry out. In recent years, as the advances of the production technology, the competition between the manufacturers is increasing, which makes the retailer more important in the supply system. Additionally, the presence of large retailers such as Wal-mart, Carrefour, and the online retailers such as Amazon and JD.com has increased the importance of the retailers in the supply chain and changed the channel structure of the supply chain. The manufacturers have to rely on large retailers for their distribution channel; thus, the retailers are dominant. Therefore, within a supply chain, either the manufacturers or the retailers can be dominant, or comparable. To this end, what is the impact on used-product collecting and which is the optimal collecting mode under such circumstances? To answer these questions, this study employs the dynamic collecting model to research this problem under different supply chain power structures.

There are increasing numbers of studies focusing on the closed-loop supply chain operation. We refer to Govindan et al. [5] for a comprehensive review on closed-loop supply chain management. The main focus of the researchers is on the collecting strategy of the supply chain members as well as the optimal reverse channel across the supply chain. In 2004, Savaskan et al. [1] first set up the reverse channel design framework from the perspective of the supply chain. Their results indicated the retailer-collecting model is preferred compared to manufacturer- and third-party-collecting models. Savaskan and Wassenhove [2] further investigated the reverse channel design problem of competing retailers. Savaskan's series of papers provided a classic framework to investigate the reverse channel design problem of closed-loop supply chain, and many other scholars have further extended their model to more complicated scenarios (Hong and Yeh [6], Huang and Nie [7], Wei and Zhao [8], Ma et al. [9]). Recently, some other studies have focused on the supply chain network design or recycle decisions. For example, Tsao et al. [10] proposed a closed-loop supply chain network design considering RFID adoption. Jena et al. [11] investigated the impact of sharing or not sharing advertisement cost on the used-products collecting activities. Bhattacharya et al. [12] investigated the used-products price optimization problem in a closed-loop supply chain system. These studies are mainly focused on the reverse channel design problem in different scenarios but have ignored the dynamic characteristics of the used-product collecting process. The retailers who aim to collect the used products must cope with the dynamic characteristics.

The above studies have significantly enriched the theoretical results on the closed-loop supply chain, yet they were all based on static model, which did not consider the dynamic characteristics of the collection process. In the dynamic collection model, Kiesmüller [13] investigated a dynamic collecting system with lead times, and applied the maximum principle to resolve the optimal control strategies of the system. Hosoda and Disney [14] also investigated the dynamics of a closed-loop supply chain with arbitrary lead times and a proportional random yield in the triage of returns. Geyer et al. [15] studied the dynamic remanufacturing strategy considering the components durability and limited life-cycle. These studies mainly concern on the dynamic feature of the inventory characteristics, and focus on centralized supply chain structure which handles the overall optimization problem of a system. These studies mainly focused on the inventory dynamic characteristics and ignored the dynamics in the collection process.

The activities which have been invested to collect the used-products have their dynamic characteristics, such as marketing type return advertising to increase the environmental awareness of customers as well as the knowledge about return policy [16] and operational type building recycling facilities, collecting, reprocessing and so on $[17,18]$. Both marketing type activities and the operational activities have long term and accumulative effects, which means the return rate of the used-product 
reflects the accumulative invest history. Consequently, the dynamic optimal control model could be applied to resolve the collecting problem.

De Giovanni and Zaccour [18] proposed a dynamic return model which utilized differential game methods to characterize the feedback equilibrium of the closed-loop supply chain with one manufacturer and one retailer. A cost-revenue sharing contract is proposed, and they found the retailer who acts as the leader always prefers the contract while the manufacturer only prefers it under particular conditions. Huang and Nie [7] also proposed a dynamic closed-loop supply chain model which captured the dynamic characteristics of the collecting process. They derived the open-loop control strategies of both the manufacturer and the retailer in a manufacturer lead Stackelberg game. Huang et al. [19] developed the dynamic model and proposed a stochastic differential game model to investigate the uncertainty factors which impact the collection strategy of a remanufacturing closed-loop supply chain [20]. These studies mainly considered the optimal control strategy problem in the supply chain where the manufacturer leads the channel.

Our research differs from the literature in the area of closed-loop supply chains in two aspects. The first is that we consider the dynamic process in the collection activities. The basis of a remanufacturing closed-loop supply chain is the collection of used-products, without which the remanufacturing of new products is out of the question. However, most researches utilized the static model to model the collection process which ignores the dynamic character of the activities. The second one is that we consider the impact of supply chain channel power structure on the closed-loop supply chain. The presence of powerful retailer has become a phenomenon in practice. How it would affect the collection activities for the closed-loop supply chain is an important question.

Thus, utilizing the dynamic collection model, this paper considered three different channel power structures to further study the optimal closed-loop supply channel collecting strategy, i.e., no channel leader, the manufacturer as the leader, the retailer as the leader. Furthermore, we discussed the effect of the different channel power structures on the optimal collecting strategy [21-28].

The rest of the paper is organized as follows. Section 2 presents the modeling framework. Section 3 derived the open-loop control strategy, respectively, for three models (N, M and R models). Section 4 conducts the comparison analysis for the three models. Section 5 concludes the paper.

\section{The Model}

The notations throughout the paper are summarized in Table 1.

Table 1. Notations throughout the paper.

\begin{tabular}{cc}
\hline Notation & Definition \\
\hline$\rho$ & The impact coefficient of collect effort on return rate. \\
$\phi$ & The deprecation ratio of the return rate. \\
$\beta$ & The market volume for the product. \\
$c_{m}, c_{r}$ & The price sensitivity coefficient. \\
$\Delta$ & The unit production cost with new raw material and used-product. \\
$b$ & The unit cost savings of used-product. \\
$r$ & The transfer price pay by manufacturer to retailer. \\
$\tau(t)$ & The collecting cost coefficient of the retailer. \\
$A(t)$ & The discount rate for manufacturer and retailer. \\
$p(t)$ & The return rate of used-product at time $t$. \\
$D(t)$ & The collect effort level at time $t$. \\
$w(t)$ & The product price at time $t$. \\
$\mu_{i}(i=m, r)$ & The demand rate at time $t$. \\
$\pi_{i}(t)(i=m, r)$ & The wholesale price at time $t$. \\
$J_{i}(i=m, r)$ & Costate variables. \\
$H_{i}(i=m, r)$ & The cumulative profit in the planning interval of manufacturer and retailer. \\
\hline
\end{tabular}


The activities which have been put into the used-product collecting process, such as return advertising, recycling facilities building and maintaining, and technology for reprocessing, all have long-term and accumulative effects, which means the return rate of the used-product reflects the accumulative invest history. Following the dynamic return model of Huang and Nie [7], Huang et al. [19], we consider dynamic characteristics in the recycling process and define the return rate variation function as

$$
\frac{d \tau(t)}{d t}=\rho A(t)-\delta \tau(t), \tau(0)=\tau_{0} \geq 0
$$

$\tau(t)<1$ represents the used-product return rate at time $t$, which is the system state variable. $A(t)$ indicates the collect effort level at time $t$, which represents the efforts made by the retailer in the collecting activities, such as cost of recycling facilities, maintenance and advertising. $\rho$ shows the effect of collect effort on return rate, and the larger of $\rho$, the faster change of return rate. $\delta$ indicates the deprecation ratio of the return rate. Equation (1) clearly depicts the dynamic process of the return rate with collecting effort of the retailer, which suppose that the return rate has a cumulative effect and is formulated as a continuous and weighted average of past return activities, with a decaying weighting function.

The manufacturer manufactures the product and passes it to the retailer for sale, the demand rate of the product is given by

$$
D(p(t))=\phi-\beta p(t)
$$

$\phi>0$ represents market volume, $\beta>0$ indicates the price sensitivity coefficient. The product either produced by recycling material or new raw material are exactly the same. What should be noted is that it is more suitable for the case the manufacturer-made use of the used-product for new production instead of just refurbish the used-product.

The unit production cost with new raw material is $c_{m}$, while the unit production cost with recycling material is $c_{r}$, and $\Delta=c_{m}-c_{r}>0$, which indicates the remanufacturing must be cost saving otherwise the manufacturer would not employ it. The manufacturer would pay $b$ to the retailer for collecting the used-product back, and we have $b \leq \Delta$. When the return transfer payment through retailer is greater than the total cost saving, the manufacturer would not choose to collect used-products for remanufacturing.

The cost function of the retailer for collecting the used-products is $k A(t)^{2} / 2$, where $k$ represent the collecting cost coefficient. In reality used-product may located in any place of retailer's distribution zone, thus, the retailer needs to perform advertising of their collection policy and to set collection facility over the entire distribution zone. As a result, the retailer may experience a high cost coefficient.

The retailer pays $\sigma$ to the customers who return back the used-product for compensation. For simplicity, we set $\sigma=0$, which should be noted that this assumption only simplified the mathematical complexity and will not change the main results.

The decision period for the members is $[0, \infty)$, and the discount rate is $r$.

We carry out the analysis in the two-stage supply chain with the retailer taking charge of the collection activities. The retailer simultaneously decides the retailer price $p(t)$ and the collect effort level $A(t)$. The objective function for the retailer is given below

$$
\max _{p(t), A(t)}\left\{J_{r}=\int_{0}^{\infty} e^{-r t}\left[(p(t)-w(t)+b \tau(t))(\phi-\beta p(t))-\frac{1}{2} k A(t)^{2}\right] d t\right\}
$$

The manufacturer decide the wholesale price $w(t)$ to the retailer, and its objective function is given by

$$
\max _{w(t)}\left\{J_{m}=\int_{0}^{\infty} e^{-r t}\left[\left(w(t)-c_{m}+(\Delta-b) \tau(t)\right)(\phi-\beta p(t))\right] d t\right\}
$$


The constraint is Equation (1). According to the power structure of the supply chain members, there are three kinds of supply chain structure, i.e., no channel leader (model N), manufacturer as channel leader (model M), and retailer as channel leader (model R). In the next section, we apply the differential game theory to solve the open-loop control strategy [29-35].

\section{Model Analysis}

We utilize the open-loop control strategy method for solving the differential game formulated in Section 2. One can refer to Sethi and Thompson [20] and Nair and Narasimhan [36] for details about the open-loop control strategy method for solving the differential games. We derive the equilibrium solution by constructing the Hamiltonians for the supply chain members [37]. Denote $\mu_{m}$ and $\mu_{r}$ to be the costate variable for the manufacturer and retailer. The current value Hamiltonian for the retailer is formulated by

$$
H_{r}\left(p, w, A, \tau, \mu_{r}\right)=(p-w+b \tau)(\phi-\beta p)-\frac{1}{2} k A^{2}+\mu_{r}(\rho A-\delta \tau)
$$

And the current value Hamiltonian for the manufacturer is given by

$$
H_{m}=\left(w-c_{m}+(\Delta-b) \tau\right)(\phi-\beta p)+\mu_{m}(\rho A-\delta \tau)
$$

\subsection{Nash Differential Game-Model N}

In such a scenario, the manufacturer and the retailer have equal channel power; thus, there are no channel leaders in the supply chain and the game they play is a Nash differential game. The decision sequence for the Nash differential game is as follows: both the manufacturer and the retailer choose their decision separately and simultaneously. The manufacturer decides the wholesale price $w(t)$, the retailer decides the retail price $p(t)$ as well as the collection effort level $A(t)$. According to retailer's current value Hamiltonian (5), the necessary conditions for retailer's optimization problem is given by

$$
\begin{gathered}
\frac{\partial H_{r}}{\partial p}=-2 \beta p-\beta(-w+b \tau)+\phi=0 \\
\frac{\partial H_{r}}{\partial A}=-k A+\mu_{r} \rho=0 \\
\frac{\partial H_{r}}{\partial \mu_{r}}=\rho A-\delta \tau \\
r \mu_{r}-\frac{\partial H_{r}}{\partial \tau}=(r+\delta) \mu_{r}-b(\phi-\beta p)
\end{gathered}
$$

Inserting $p=m+w$ into manufacturer's current value Hamiltonian (6) we have

$$
H_{m}=\left(w-c_{m}+(\Delta-b) \tau\right)(\phi-\beta p)+\mu_{m}(\rho A-\delta \tau)
$$

The necessary conditions for manufacturer's optimization problem are given by

$$
\begin{gathered}
\frac{\partial H_{m}}{\partial w}=\phi-\beta p-\beta\left(w-c_{m}+\Delta \tau\right)+b \beta \tau=0 \\
\dot{\mu}_{m}=r \mu_{m}-\frac{\partial H_{m}}{\partial \tau}=(r+\delta) \mu_{m}-\Delta(\phi-\beta p)-b(\phi-\beta p)
\end{gathered}
$$

From Equations (7) and (12) can derive the equilibrium strategies of manufacturer and retailer

$$
w^{*}=\frac{\phi+2 \beta c_{m}-2 \beta \Delta \tau+3 b \beta \tau}{3 \beta}, p^{*}=\frac{2 \phi+\beta c_{m}-\beta \Delta \tau}{3 \beta}
$$


And we can derive the equilibrium collecting effort strategy from Equation (8)

$$
A^{*}=\frac{\rho}{k} \mu_{r}
$$

Substituting the equilibrium strategies into Equations (9) and (10) yields the differential equations about the state variable $\tau$ and the costate variable $\mu_{r}$ as below

$$
\left\{\begin{array}{c}
\dot{\tau}=-\delta+\frac{\rho^{2}}{k} \mu_{r} \\
\dot{\mu}_{r}=E_{1} \tau+(r+\delta) \mu_{r}+F_{1}
\end{array}\right.
$$

where $E_{1}=-\beta \Delta b / 3$ and $F_{1}=-b\left(\phi-\beta c_{m}\right) / 3$. Proposition 1 gives the condition under which the steady equilibrium exists in the Nash setting.

Proposition 1. When $k \delta(r+\delta)>-\rho^{2}\left(E_{1}+F_{1}\right)$, the steady equilibrium of Model $N$ exists and the steady equilibrium is given by

$$
\bar{\tau}^{N}=\frac{-\rho^{2} F_{1}}{k \delta(r+\delta)+\rho^{2} E_{1}}, \bar{\mu}_{r}^{N}=\frac{-\delta k F_{1}}{k \delta(r+\delta)+\rho^{2} E_{1}}
$$

The wholesale price of the manufacturer when the system is steady state is calculated as

$$
\bar{w}^{N}=\frac{\phi+2 \beta c_{m}}{3 \beta}+\frac{(3 b-2 \Delta)}{3} \bar{\tau}^{N}
$$

The retail price and collecting effort level of the retailer are calculated as

$$
\bar{p}^{N}=\frac{2 \phi+\beta c_{m}}{3 \beta}-\frac{\Delta}{3} \bar{\tau}^{N}, \bar{A}^{N}=\frac{\rho}{k} \bar{\mu}_{r}^{N}
$$

Proof. The steady state of return rate and costate variable can be obtained by solving the differential Equation (16). The characteristic root of differential Equation (16) is calculated by

$$
\lambda_{1,2}=\frac{r k \pm \sqrt{k^{2} r^{2}+4 k^{2} \delta r+4 k^{2} \delta^{2}+4 k \rho^{2} E_{1}}}{2 k}
$$

As long as the equations have real characteristic root, we always have $\lambda_{1}>0$. Thus, when the differential equations have convergence solution, it will be a saddle point equilibrium. When $4 k^{2} \delta r+$ $4 k^{2} \delta^{2}+4 k \rho^{2} E_{1}<0$, it is obvious that $\lambda_{2}>0$, and in this case the solution of differential equations is divergence. When $4 k^{2} \delta r+4 k^{2} \delta^{2}+4 k \rho^{2} E_{1}>0$, it is obvious that $\lambda_{2}<0$, and in this case the solution of the differential equations is saddle point equilibrium, which means when $k \delta(r+\delta)>-\rho^{2} E_{1}$, there is steady state equilibrium for the system. We can derive the steady state of the system from the differential equations by $\dot{\tau}=0$ and $\dot{\mu}_{r}=0$. And in addition, as the return rate of the system cannot exceed 1 , thus the steady state of return rate should satisfy $\bar{\tau}<1$, i.e., $k \delta(r+\delta)+\rho^{2} E_{1}>-\rho^{2} F_{1}$, simplified as $k \delta(r+\delta)>-\rho^{2}\left(E_{1}+F_{1}\right)$. As a result, $F_{1}<0$, it is obvious that when $k \delta(r+\delta)>$ $-\rho^{2}\left(E_{1}+F_{1}\right)$ we can always have $k \delta(r+\delta)>-\rho^{2} E_{1}$. The proof is complete.

Proposition 1 indicates if retailer experiencing a relatively high collection cost coefficient, the system will reach a steady state, otherwise when the cost coefficient is small, the system will be unstable. Furthermore, when the cost coefficient is too small, the retailer has incentive to collect all the used-product. However, the distribution of used product is relatively dispersed and combined with high transportation expense, thus it is very unlikely for retailer to collect all the used-product. Consequently, in reality, the collection cost coefficient is reality large, which could satisfy the stable 
equilibrium condition of the differential equations. Substitute the steady state results of Proposition 1 into profit function of supply chain member, we have the steady state profit rate of retailer.

$$
\bar{\pi}_{r}^{N}=\frac{\left(\phi-\beta c_{m}+\beta \Delta \bar{\tau}^{N}\right)^{2}}{9 \beta}-\frac{\rho^{2}}{2 k}\left(\bar{\mu}_{r}^{N}\right)^{2}
$$

And the steady state profit rate of manufacturer

$$
\bar{\pi}_{m}^{N}=\frac{\left(\phi-\beta c_{m}+\beta \Delta \bar{\tau}^{N}\right)^{2}}{9 \beta}
$$

The following proposition characterizes the open-loop equilibrium control strategies for the supply chain members.

Proposition 2. Under the setting of Nash differential game (Model N), the optimal wholesale price of manufacturer is given by

$$
w^{N}(t)=\frac{\phi+2 \beta c_{m}}{3 \beta}+\frac{(3 b-2 \Delta)\left(\tau_{0}-\bar{\tau}^{N}\right) e^{\lambda_{2} t}}{3}+\frac{(3 b-2 \Delta)}{3} \bar{\tau}^{N}
$$

The optimal collecting effort control strategy of retailer is

$$
A^{N}(t)=\frac{\delta+\lambda_{2}}{\rho}\left(\tau_{0}-\bar{\tau}^{N}\right) e^{\lambda_{2} t}+\frac{\rho}{k} \bar{\mu}_{r}^{N}
$$

The optimal retail price control strategy of retailer is

$$
p^{N}(t)=\frac{2 \phi+\beta c_{m}}{3 \beta}-\frac{\Delta\left(\tau_{0}-\bar{\tau}^{N}\right) e^{\lambda_{2} t}}{3}-\frac{\Delta}{3} \bar{\tau}^{N}
$$

Proof. Equation (16) is differential equations, as the problem we consider in this paper is infinite time limit, let the terminal condition as $\lim _{T \rightarrow \infty} \tau(t)=\bar{\tau}^{N}$, where $\bar{\tau}^{N}$ is the steady state of the state variable. Combining the initial condition $\tau(0)=\tau_{0}$, the solution of the differential equations is solved as

$$
\left\{\begin{array}{c}
\tau^{N}(t)=\left(\tau_{0}-\bar{\tau}^{N}\right) e^{\lambda_{2} t}+\bar{\tau}^{N} \\
\mu_{r}{ }^{N}(t)=\frac{k\left(\delta+\lambda_{2}\right)\left(\tau_{0}-\bar{\tau}^{N}\right) e^{\lambda_{2} t}}{\rho^{2}}+\bar{\mu}_{r}^{N}
\end{array}\right.
$$

Substituting Equation (26) into the equilibrium control strategies of the supply chain members can yield the optimal control strategies in Proposition 2. The proof is complete.

\subsection{Manufacturer as Leader Stackelberg Differential Game-Model M}

In this scenario, manufacturer is the channel leader in the supply chain and retailer be as the follower, thus the game is a manufacturer leader Stackelberg differential game. The decision sequence of Model M is as follows: the manufacturer first decides the wholesale price $w(t)$, and then the retailer chooses its retail price $p(t)$ and collecting effort level $A(t)$. The necessary conditions are still Equations (7)-(10), and from which can derive the response function of retailer

$$
p^{*}=\frac{\phi+\beta w-b \beta \tau}{2 \beta}, A^{*}=\frac{\rho}{k} \mu_{r}
$$


According to the retailer's response function, the current value Hamiltonian of the manufacturer could be formulated as

$$
H_{m}=\left(w-c_{m}+(\Delta-b) \tau\right)\left(\phi-\beta p^{*}\right)+\mu_{m}\left(\rho A^{*}-\delta \tau\right)
$$

According to the necessary optimality condition $\partial H_{m} / \partial w=0$, we have

$$
w^{*}=\frac{\phi+\beta c_{m}+2 b \beta \tau-\beta \Delta \tau}{2 \beta}
$$

Substituting back into the response function of retailer yields

$$
p^{*}=\frac{3 \phi+\beta c_{m}-\beta \Delta \tau}{4 \beta}, A^{*}=\frac{\rho}{k} \mu_{r}
$$

Substituting the equilibrium strategies of manufacturer and retailer into Equations (9) and (10) yields the differential equations about the state variable $\tau$ and the costate variable $\mu_{r}$ as follows

$$
\left\{\begin{array}{c}
\dot{\tau}=-\delta+\frac{\rho^{2}}{k} \mu_{r} \\
\dot{\mu}_{r}=X_{1} \tau+(r+\delta) \mu_{r}+Y_{1}
\end{array}\right.
$$

where $X_{1}=-\beta \Delta b / 4$ and $Y_{1}=-b\left(\phi-\beta c_{m}\right) / 4$. Proposition 3 gives the condition under which the steady equilibrium exists in the manufacturer leader Stackelberg game setting.

Proposition 3. When $k \delta(r+\delta)>-\rho^{2}\left(X_{1}+Y_{1}\right)$, the steady equilibrium of Model M exists and the steady equilibrium is given by

$$
\bar{\tau}^{M}=\frac{-\rho^{2} Y_{1}}{k \delta(r+\delta)+\rho^{2} X_{1}}, \bar{\mu}_{r}^{M}=\frac{-\delta k Y_{1}}{k \delta(r+\delta)+\rho^{2} X_{1}}
$$

The wholesale price of the manufacturer when the system is steady state is

$$
\bar{w}^{M}=\frac{\phi+\beta c_{m}}{2 \beta}-\frac{(\Delta-2 b)}{2} \bar{\tau}^{M}
$$

And the retail price and collecting effort level of the retailer are

$$
\bar{p}^{M}=\frac{3 \phi+\beta c_{m}}{4 \beta}-\frac{\Delta}{4} \bar{\tau}^{M}, \bar{A}^{M}=\frac{\rho}{k} \bar{\mu}_{r}^{M}
$$

Proof. The proof is similar to that of Proposition 1 and thus we omit here.

Proposition 3 also indicates that the system reaches a steady state only with the high collection cost coefficient, the supply chain is under the lead of manufacturer. Likewise, we have the steady state profit rate of retailer.

$$
\bar{\pi}_{r}^{M}=\frac{\left(\phi-\beta c_{m}+\beta \Delta \bar{\tau}^{M}\right)^{2}}{16 \beta}-\frac{\rho^{2}}{2 k}\left(\bar{\mu}_{r}^{M}\right)^{2}
$$

And the steady state profit rate of manufacturer is

$$
\bar{\pi}_{m}^{M}=\frac{\left(\phi-\beta c_{m}+\beta \Delta \bar{\tau}^{M}\right)^{2}}{8 \beta}
$$

Proposition 4 gives the open-loop equilibrium control strategies for the supply chain members of model M. 
Proposition 4. Under the setting of manufacturer leader Stackelberg differential game (Model M), the optimal wholesale price of manufacturer is given by

$$
w^{M}(t)=\frac{\phi+\beta c_{m}}{2 \beta}-\frac{(\Delta-2 b)\left(\tau_{0}-\bar{\tau}^{M}\right) e^{\alpha_{2} t}}{2}-\frac{(\Delta-2 b)}{2} \bar{\tau}^{M}
$$

The optimal collecting effort control strategy of retailer is calculated as

$$
A^{M}(t)=\frac{\delta+\alpha_{2}}{\rho}\left(\tau_{0}-\bar{\tau}^{M}\right) e^{\alpha_{2} t}+\frac{\rho}{k} \bar{\mu}_{r}^{M}
$$

And the optimal retail price control strategy of retailer is

$$
p^{M}(t)=\frac{3 \phi+\beta c_{m}}{4 \beta}-\frac{\Delta\left(\tau_{0}-\bar{\tau}^{M}\right) e^{\alpha_{2} t}}{4}-\frac{\Delta}{4} \bar{\tau}^{M}
$$

Proof. The proof is similar to that of Proposition 2 and, thus, we omit here.

\subsection{Retailer as Leader Stackelberg Differential Game-Model $R$}

In this scenario, the retailer is the channel leader in the supply chain and the manufacturer be as the follower, thus, the game is a retailer leader Stackelberg differential game. The decision sequence of Model $\mathrm{R}$ is as follows: the retailer first decides the retail price $p(t)$ and the collecting effort level $A(t)$, and then the manufacturer decides the wholesale price $w(t)$. According to the backward induction, the current value Hamilton of manufacturer is formulated as Equation (11), and from the necessary optimality condition (12) can derive the response function of the manufacturer as following

$$
w^{*}=\frac{\phi-\beta p+\beta c_{m}-\beta(\Delta-b) \tau}{\beta}
$$

Considering manufacturer's response function, the current value Hamilton of retailer is formulated as

$$
H_{r}=\left(p-w^{*}+b \tau\right)(\phi-\beta p)-\frac{1}{2} k A^{2}+\mu_{r}(\rho A-\delta \tau)
$$

The necessary optimality conditions are given by

$$
\begin{gathered}
\frac{\partial H_{r}}{\partial p}=-4 \beta p+3 \phi+\beta c_{m}-\beta \Delta \tau=0 \\
\frac{\partial H_{r}}{\partial A}=-k A+\mu_{r} \rho=0 \\
\frac{\partial H_{r}}{\partial \mu_{r}}=\dot{\tau}=\rho A-\delta \tau \\
\dot{\mu}_{r}=r \mu_{r}-\frac{\partial H_{r}}{\partial \tau}=(r+\delta) \mu_{r}-\Delta(\phi-\beta p)
\end{gathered}
$$

According to Equations (42) and (43) can yield the equilibrium strategies of retailer

$$
p^{*}=\frac{3 \phi+\beta c_{m}-\beta \Delta \tau}{4 \beta}, A^{*}=\frac{\rho}{k} \mu_{r}
$$


Taking back into the response function of the manufacturer yields the equilibrium strategy of manufacturer

$$
w^{*}=\frac{\phi+3 \beta c_{m}-3 \beta \Delta \tau+4 \beta b \tau}{4 \beta}
$$

Substituting the equilibrium strategies of manufacturer and retailer into Equations (44) and (45) yields the differential equations about the state variable $\tau$ and the costate variable $\mu_{r}$ as follows

$$
\left\{\begin{array}{c}
\dot{\tau}=-\delta+\frac{\rho^{2}}{k} \mu_{r} \\
\dot{\mu}_{r}=M_{1} \tau+(r+\delta) \mu_{r}+N_{1}
\end{array}\right.
$$

where $M_{1}=-\Delta^{2} \beta / 4$ and $N_{1}=-\Delta\left(\phi-\beta c_{m}\right) / 4$. Proposition 5 gives the condition under which the steady equilibrium exists in the retailer leader Stackelberg game setting.

Proposition 5. When $k \delta(r+\delta)>-\rho^{2}\left(M_{1}+N_{1}\right)$, the steady equilibrium of Model R exists and the steady equilibrium is given by

$$
\bar{\tau}^{R}=\frac{-\rho^{2} N_{1}}{k \delta(r+\delta)+\rho^{2} M_{1}}, \bar{\mu}_{r}^{R}=\frac{-\delta k N_{1}}{k \delta(r+\delta)+\rho^{2} M_{1}}
$$

The wholesale price of the manufacturer when the system is steady state is

$$
\bar{w}^{R}=\frac{\phi+3 \beta c_{m}}{4 \beta}-\frac{(3 \Delta-4 b)}{4} \bar{\tau}^{R}
$$

And the retail price and collecting effort level of the retailer are

$$
\bar{p}^{R}=\frac{3 \phi+\beta c_{m}}{4 \beta}-\frac{\Delta}{4} \bar{\tau}^{R}, \bar{A}^{R}=\frac{\rho}{k} \bar{\mu}_{r}^{R}
$$

Proof. The proof is similar to that of Proposition 1 and thus we omit here.

Substitute the steady state results of Proposition 5 into profit function of supply chain member, we have the steady state profit rate of retailer.

$$
\bar{\pi}_{r}^{R}=\frac{\left(\phi-\beta c_{m}+\beta \Delta \bar{\tau}^{R}\right)^{2}}{8 \beta}-\frac{\rho^{2}}{2 k}\left(\bar{\mu}_{r}^{R}\right)^{2}
$$

And also the steady state profit rate of manufacturer

$$
\bar{\pi}_{m}^{R}=\frac{\left(\phi-\beta c_{m}+\beta \Delta \bar{\tau}^{R}\right)^{2}}{16 \beta}
$$

The following proposition 6 gives the open-loop equilibrium control strategies for the supply chain members of model R.

Proposition 6. Under the setting of retailer leader Stackelberg differential game (Model R), the optimal wholesale price of manufacturer is given by

$$
w^{R}(t)=\frac{\phi+3 \beta c_{m}}{4 \beta}-\frac{(3 \Delta-4 b)\left(\tau_{0}-\bar{\tau}^{R}\right) e^{\xi_{2} t}}{4}-\frac{(3 \Delta-4 b)}{4} \bar{\tau}^{R}
$$


The optimal collecting effort control strategy of retailer is calculated as

$$
A^{R}(t)=\frac{\delta+\xi_{2}}{\rho}\left(\tau_{0}-\bar{\tau}^{R}\right) e^{\xi_{2} t}+\frac{\rho}{k} \bar{\mu}_{r}^{R}
$$

And the optimal retail price control strategy of retailer is

$$
p^{R}(t)=\frac{3 \phi+\beta c_{m}}{4 \beta}-\frac{\Delta\left(\tau_{0}-\bar{\tau}^{R}\right) e^{\tilde{\zeta}_{2} t}}{4}-\frac{\Delta}{4} \bar{\tau}^{R}
$$

Proof. The proof is similar to that of Proposition 2 and thus we omit here. $\square$

\section{Comparison Analysis under Different Models}

Sections 3.1-3.3 analyze the equilibrium control strategies for the supply chain members with no channel leader (model $\mathrm{N}$ ), manufacturer as channel leader (model $\mathrm{M}$ ), and retailer as channel leader (model R), respectively. In this section, we will conduct the comparison analysis on three models to study how the presence of the power retailer would affect the system price, product collecting rate as well as member profitability. We assume that all three models can reach steady state equilibrium conditions.

Proposition 7. When $b \in(0,3 \Delta / 4), \bar{\tau}^{R}>\bar{\tau}^{N}>\bar{\tau}^{M} ;$ When $b \in(3 \Delta / 4, \Delta], \bar{\tau}^{N}>\bar{\tau}^{R}>\bar{\tau}^{M}$.

Proof. From the expressions of $\bar{\tau}^{R}$ and $\bar{\tau}^{M}$, it is calculated as

$$
\bar{\tau}^{R}-\bar{\tau}^{M}=\frac{4 \rho^{2} k \delta\left(\phi-\beta c_{m}\right)(r+\delta)(\Delta-b)}{\left[4 k \delta(r+\delta)-\rho^{2} \Delta^{2} \beta\right]\left[4 k \delta(r+\delta)-\rho^{2} \Delta b \beta\right]}>0
$$

Similarly from $\bar{\tau}^{N}$ and $\bar{\tau}^{M}$, we have

$$
\bar{\tau}^{N}-\bar{\tau}^{M}=\frac{\rho^{2} k \delta b\left(\phi-\beta c_{m}\right)(r+\delta)}{\left[3 k \delta(r+\delta)-\rho^{2} \Delta b \beta\right]\left[4 k \delta(r+\delta)-\rho^{2} \Delta b \beta\right]}>0
$$

And from $\bar{\tau}^{N}$ and $\bar{\tau}^{R}$, we have

$$
\bar{\tau}^{N}-\bar{\tau}^{R}=\frac{\rho^{2} k \delta\left(\phi-\beta c_{m}\right)(r+\delta)(4 b-3 \Delta)}{\left[3 k \delta(r+\delta)-\rho^{2} \Delta b \beta\right]\left[4 k \delta(r+\delta)-\rho^{2} \Delta^{2} \beta\right]}
$$

It is obvious that when $4 b-3 \Delta>0$, that is when $b>3 \Delta / 4, \bar{\tau}^{N}>\bar{\tau}^{R}$; vice-versa when $b<3 \Delta / 4$, $\bar{\tau}^{R}>\bar{\tau}^{N}$. The proof is complete.

Proposition 7 indicates that when in the steady state, the manufacturer acts as channel leader and this would result in the lowest return rate compared with the other two models. Regarding the retailer leader setting and no leader setting, when the transfer price of used-product is relatively high $(b>3 \Delta / 4)$, model $\mathrm{N}$ (no channel leader) would result in higher return rate. Furthermore, when the transfer price of used-product is not that high $(b<3 \Delta / 4)$, model $\mathrm{R}$ (retailer as channel leader) would result in higher return rate. The results indicate that when the manufacturer leads the channel, the retailer has the lowest incentive to collect in a dynamic return model. As the transfer price is high, the collecting efficiency of the retailer is higher under the scenario where there is no leader in the supply chain. However, when the transfer price is low, the retailer will have an incentive to collect only if the retailer were channel leader. 
Proposition 8. When $b \in(0,3 \Delta / 4), \bar{A}^{R}>\bar{A}^{N}>\bar{A}^{M} ;$ When $b \in(3 \Delta / 4, \Delta], \bar{A}^{N}>\bar{A}^{R}>\bar{A}^{M}$.

Proof. Compare with $\bar{A}^{R}, \bar{A}^{N}$ and $\bar{A}^{M}$ yield

$$
\begin{gathered}
\bar{A}^{R}-\bar{A}^{M}=\frac{4 k^{2} \delta^{2}\left(\phi-\beta c_{m}\right)(r+\delta)(\Delta-b)}{\left[4 k \delta(r+\delta)-\rho^{2} \Delta^{2} \beta\right]\left[4 k \delta(r+\delta)-\rho^{2} \Delta b \beta\right]}>0 \\
\bar{A}^{N}-\bar{A}^{M}=\frac{k^{2} \delta^{2} b\left(\phi-\beta c_{m}\right)(r+\delta)}{\left[3 k \delta(r+\delta)-\rho^{2} \Delta b \beta\right]\left[4 k \delta(r+\delta)-\rho^{2} \Delta b \beta\right]}>0 \\
\bar{A}^{N}-\bar{A}^{R}=\frac{k^{2} \delta^{2}\left(\phi-\beta c_{m}\right)(r+\delta)(4 b-3 \Delta)}{\left[3 k \delta(r+\delta)-\rho^{2} \Delta b \beta\right]\left[4 k \delta(r+\delta)-\rho^{2} \Delta^{2} \beta\right]}
\end{gathered}
$$

It is obvious to derive the conclusion in Proposition 8. The proof is complete.

Proposition 8 indicates, when the transfer price of used-product is high $(b>3 \Delta / 4)$, the retailer will put more effort into collecting activities in the setting that there is no channel leader. When the transfer price is not that attractive $(b<3 \Delta / 4)$, the retailer will put more collecting effort only when it leads the channel.

Proposition 9. When $b<\left[\rho^{2} \Delta^{2} \beta-k \delta(r+\delta)\right] /\left(\rho^{2} \beta \Delta\right), \bar{p}^{R}<\bar{p}^{N}<\bar{p}^{M}$; When $b>$ $\left[\rho^{2} \Delta^{2} \beta-k \delta(r+\delta)\right] /\left(\rho^{2} \beta \Delta\right), \bar{p}^{N}<\bar{p}^{R}<\bar{p}^{M}$.

Proof. Compare with $\bar{p}^{R}, \bar{p}^{N}$ and $\bar{p}^{M}$ yield

$$
\begin{gathered}
\bar{p}^{R}-\bar{p}^{M}=\frac{-\Delta \rho^{2} k \delta\left(\phi-\beta c_{m}\right)(r+\delta)(\Delta-b)}{\left[4 k \delta(r+\delta)-\rho^{2} \Delta^{2} \beta\right]\left[4 k \delta(r+\delta)-\rho^{2} \Delta b \beta\right]}<0 \\
\bar{p}^{N}-\bar{p}^{M}=\frac{-k^{2} \delta^{2}\left(\phi-\beta c_{m}\right)(r+\delta)^{2}}{\beta\left[3 k \delta(r+\delta)-\rho^{2} \Delta b \beta\right]\left[4 k \delta(r+\delta)-\rho^{2} \Delta b \beta\right]}<0 \\
\bar{p}^{N}-\bar{p}^{R}=\frac{-k \delta\left(\phi-\beta c_{m}\right)(r+\delta)\left(k \delta(r+\delta)-\rho^{2} \Delta^{2} \beta+\rho^{2} \beta \Delta b\right)}{\beta\left[3 k \delta(r+\delta)-\rho^{2} \Delta b \beta\right]\left[4 k \delta(r+\delta)-\rho^{2} \Delta^{2} \beta\right]}<0
\end{gathered}
$$

Thus, when $k \delta(r+\delta)-\rho^{2} \Delta^{2} \beta+\rho^{2} \beta \Delta b>0$, that is when $b>\left[\rho^{2} \Delta^{2} \beta-k \delta(r+\delta)\right] / \rho^{2} \beta \Delta$, $\bar{p}^{N}<\bar{p}^{R}$; Otherwise, when $b<\left[\rho^{2} \Delta^{2} \beta-k \delta(r+\delta)\right] / \rho^{2} \beta \Delta, \bar{p}^{N}>\bar{p}^{R}$. The proof is complete.

Proposition 9 indicates that the presence of the power retailer would lead to the lowest product market price when the transfer price of the used-product is low $\left(b<\left[\rho^{2} \Delta^{2} \beta-k \delta(r+\delta)\right] /\left(\rho^{2} \beta \Delta\right)\right)$; and when the transfer price is relatively high $\left(b>\left[\rho^{2} \Delta^{2} \beta-k \delta(r+\delta)\right] /\left(\rho^{2} \beta \Delta\right)\right)$, the product market price will be lowest under the setting of no channel leader (model $\mathrm{N}$ ). The results show that the manufacturer lead setting (model $\mathrm{M}$ ) would results in a highest product market price, which means it does not contribute to lowering of the market price which would bring about increasing of sales volume and consumer's utility.

Proposition 10. When $k \delta(r+\delta)>\rho^{2} \Delta^{2} \beta / 2, \bar{w}^{R}<\bar{w}^{N}<\bar{w}^{M}$; otherwise when $k \delta(r+\delta)<\rho^{2} \Delta^{2} \beta / 2$, there is a threshold value about transfer price $\widetilde{b}=\sqrt{2 k \delta(r+\delta) / \rho^{2} \beta}$, when $b<\widetilde{b}, \bar{w}^{R}<\bar{w}^{N}<\bar{w}^{M}$, and when $b>\widetilde{b}, \bar{w}^{R}<\bar{w}^{M}<\bar{w}^{N}$.

Proof. Compare with $\bar{w}^{R}$ and $\bar{w}^{M}$

$$
\bar{w}^{R}-\bar{w}^{M}=\frac{-k \delta\left(\phi-\beta c_{m}\right)(r+\delta)\left(4 \beta \rho^{2} b^{2}-7 \beta \Delta \rho^{2} b+4 k \delta r+4 k \delta^{2}+2 \rho^{2} \Delta^{2} \beta\right)}{\beta\left[4 k \delta(r+\delta)-\rho^{2} \Delta^{2} \beta\right]\left[4 k \delta(r+\delta)-\rho^{2} \Delta b \beta\right]}
$$


The expression $y(b)=4 \beta \rho^{2} b^{2}-7 \beta \Delta \rho^{2} b+4 k \delta r+4 k \delta^{2}+2 \rho^{2} \Delta^{2} \beta$ is a quadratic function about $b$. Its minimum value on the interval $[0, \Delta]$ is calculated by $y(7 \Delta / 8)=4 k \delta(r+\delta)-17 \rho^{2} \Delta^{2} \beta / 16$. As $k \delta(r+\delta)>\rho^{2}\left(\beta \Delta^{2}+\phi-\beta c_{m}\right) / 4$, we have $4 k \delta(r+\delta)-17 \rho^{2} \Delta^{2} \beta / 16>0$. Consequently when $b \in[0, \Delta], y(b)>0$, which means $\bar{w}^{R}<\bar{w}^{M}$.

The comparison of $\bar{w}^{M}$ and $\bar{w}^{N}$ is in the similar way. Compare with $\bar{w}^{N}$ and $\bar{w}^{M}$

$$
\bar{w}^{N}-\bar{w}^{M}=\frac{k \delta\left(\phi-\beta c_{m}\right)(r+\delta)\left(\beta \rho^{2} b^{2}-2 k \delta(r+\delta)\right)}{\beta\left[3 k \delta(r+\delta)-\rho^{2} \Delta b \beta\right]\left[4 k \delta(r+\delta)-\rho^{2} \Delta b \beta\right]}
$$

It is easily to verified when $b<\sqrt{2 k \delta(r+\delta) / \rho^{2} \beta}, \beta \rho^{2} b^{2}-2 k \delta(r+\delta)<0$; and when $b>$ $\sqrt{2 k \delta(r+\delta) / \rho^{2} \beta}, \beta \rho^{2} b^{2}-2 k \delta(r+\delta)>0$. If $\sqrt{2 k \delta(r+\delta) / \rho^{2} \beta}>\Delta$, when $b \in[0, \Delta]$, it is always true that $\beta \rho^{2} b^{2}-2 k \delta(r+\delta)<0$, which means $\bar{w}^{N}-\bar{w}^{M}<0$. If $\sqrt{2 k \delta(r+\delta) / \rho^{2} \beta}<\Delta$, there is a threshold value about transfer price that $\widetilde{b}=\sqrt{2 k \delta(r+\delta) / \rho^{2} \beta}$, when $b<\widetilde{b}, \bar{w}^{N}-\bar{w}^{M}<0$, and when $b>\widetilde{b}$, $\bar{w}^{N}-\bar{w}^{M}>0$. The proof is complete.

As the profit functions under different models are too complex to deal with by an analytical approach, we will conduct a numerical comparison instead in the following part. The parameters are chosen as: $\tau_{0}=0, \rho=2, \Delta=3, \phi=50, \beta=0.8, c_{m}=6, \delta=1, r=0.15, k=150$. The transfer price $b$ will be limited in a reasonable section as $b<\Delta$ to make the numerical results be consistent with the actual situations.

Figure 1 shows that $\pi_{r}^{R}(t)>\pi_{r}^{N}(t)$, and Figure 2 shows that $\pi_{r}^{N}(t)>\pi_{r}^{M}(t)$, consequently we can derive $\pi_{r}^{R}(t)>\pi_{r}^{N}(t)>\pi_{r}^{M}(t)$. The result is quite intuitive: the retailer prefers itself to be the leader in the channel mostly in the three settings and does not prefer the manufacturer to be the leader.

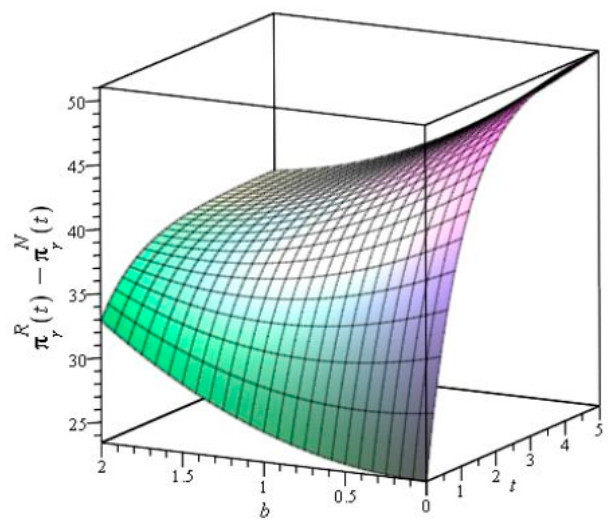

Figure 1. Retailer's profit rate difference between model $R$ and $N$.

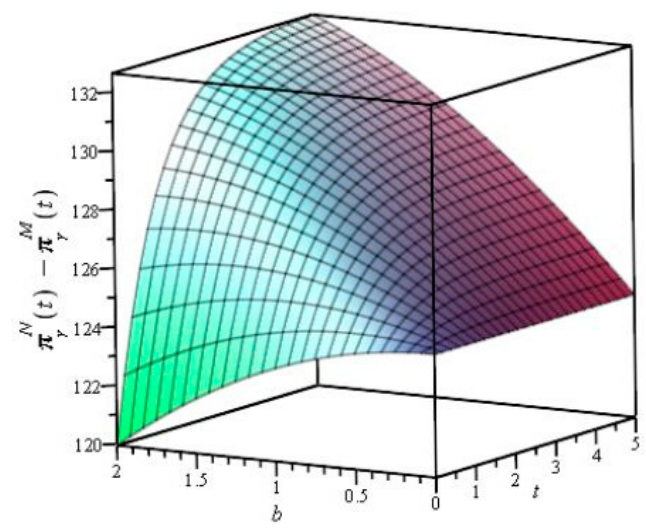

Figure 2. Retailer's profit rate difference between model $N$ and $M$. 
Figure 3 shows that $\pi_{m}^{M}(t)>\pi_{m}^{N}(t)$, and Figure 4 shows that $\pi_{m}^{N}(t)>\pi_{m}^{R}(t)$; consequently, we can derive $\pi_{m}^{M}(t)>\pi_{m}^{N}(t)>\pi_{m}^{R}(t)$. The result is quite similar to the retailer's, which means both manufacturer and retailer prefer the setting that is led by themselves while they dislike the setting that is led by other supply chain members.

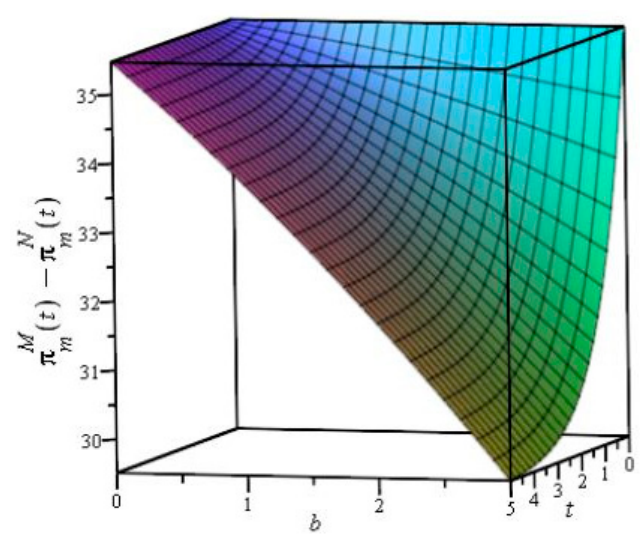

Figure 3. Manufacturer's profit rate difference between model $M$ and $N$.

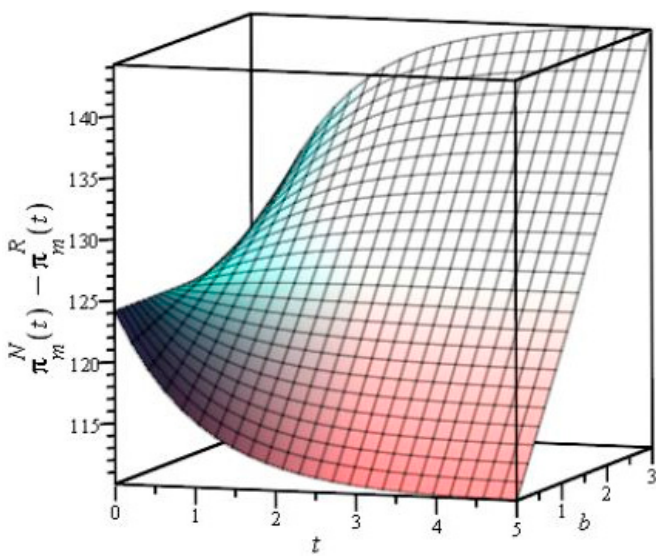

Figure 4. Manufacturer's profit rate difference between model $N$ and $R$.

In the discussion above, we have not investigated the choice of the transfer price $b$ for the manufacturer. With a higher transfer price, the retailer would have more enthusiasm to invest in collection activities, while the manufacturer would result in lower benefits from used-product.

Proposition 11. In model $N$ and model $M$, the optimal transfer price of the used-product is $b^{N *}=b^{M *}=\Delta$, while in model $R$, the optimal transfer price of the used-product is $b^{R *} \in[0, \Delta]$.

Proof. It is very easy to verify that $\partial \bar{\pi}_{m}^{N} / \partial b>0, \partial \bar{\pi}_{m}^{M} / \partial b>0$ and $\partial \bar{\pi}_{m}^{R} / \partial b=0$. And as the transfer price has a range in $[0, \Delta]$, the manufacturer could easily choose the transfer price which is given in Proposition 11. The proof is complete.

Proposition 11 indicates that the manufacturer should transfer all the unit cost savings to the retailer when the manufacturer leads the supply chain, or no one leads the supply chain. Although the manufacturer transfers all the unit cost savings to the retailer, the manufacturer could benefit from increasing product sales. It is interesting that the transfer price has no impact on the steady state profit rate of the manufacturer when the retailer leads the supply chain. That is mainly because the 
manufacturer could not affect the decision of the retailer in the presence of power retailers. Therefore, the transfer price has lost the incentive to the retailer.

Proposition 12. Let $b=\Delta$ in all the three models, $\bar{\tau}^{N}>\bar{\tau}^{R}=\bar{\tau}^{M}, \bar{A}^{N}>\bar{A}^{R}=\bar{A}^{M}, \bar{p}^{N}<\bar{p}^{R}=\bar{p}^{M}$, $\bar{w}^{R}<\bar{w}^{M}<\bar{w}^{N}, \bar{\pi}_{r}^{R}>\bar{\pi}_{r}^{N}>\bar{\pi}_{r}^{M}, \bar{\pi}_{m}^{M}>\bar{\pi}_{m}^{N}>\bar{\pi}_{m}^{R}$.

Proof. The proof is similar to that of Propositions 7-10 and thus we omit here.

When the manufacturer transfers all the unit cost savings to the retailer, the manufacturer leading the supply chain would result in the same return rate and product price. That means the manufacturers would incentive the retailer in the collection activities by transferring all the unit cost savings when they lead. Although the return rate is highest when no one leads the supply chain, the profitability is determined by channel power more than the product return efficiency. However, from a sustainability perspective, the channel power enhancement of the retailer would be beneficial to the environment with low waste emissions. The manufacturer would give away some channel power to increase the product reuse efficiency when it leads the supply chain.

\section{Conclusions}

In this study, we considered the presence of the power retailer on the dynamic collecting problem in a closed-loop supply chain, which consists of a manufacturer and a retailer who is responsible for the used-product collecting. For a closed-loop supply chain, the key procedure is the collection of the used-product which are to be used for the production of new products. The main focus of this study is the dynamic characteristics of the collecting process and the presence of the power retailer on the reverse channel. Firstly, we formulated the dynamic collecting model by the differential equation; and then we derived the equilibrium of three settings, i.e., no channel leader Nash differential game (model $N$ ), manufacturer leader Stackelberg differential game (model $M$ ), and retailer leader Stackelberg differential game (model $R$ ). Thirdly, we conducted the comparison analysis on return rate, price and profit rate in the three models.

The equilibrium results show that the collection cost coefficient must be relatively high or else the dynamic system cannot reach a steady state and will be unstable, regardless of the model setting, i.e., model $N, M$ or $R$.

The comparison results show that when the manufacturer leads the channel, the retailer has the lowest incentive to collect in a dynamic return model. That means the presence of a power retailer is beneficial to the reverse channel of the closed-loop supply chain. When the transfer price is high, the collecting efficiency of the retailer is higher under the scenario there is no leader in the supply chain. That means when the transfer price the manufacturer affords is relatively high, the retailer no longer needs the channel power. However, when the transfer price is low, the retailer will have the incentive to collect only if the retailer itself were channel leader. That means when the transfer price is unattractive, the retailer needs the channel power to ensure the benefit of the collecting activities.

To the market price, the results show that manufacturer-led setting (model $M$ ) would result in a higher product market price. Furthermore, when the transfer price is low, the retailer leader setting can lead to lowest market price, as a result, the retailer puts more efforts in collecting used-products under this scenario.

To the profit rate of manufacturer and retailer, the comparison results of three models are quite similar. The results show that both the manufacturer and the retailer prefer to be the channel leader while they dislike the other channel members to be the leaders. This means that although the presence of power retailer is beneficial to the collecting efficiency in the closed-loop supply chain, it is not enough to increase the profit level of the manufacturer. However, from a sustainable innovation perspective, the presence of power retailer would be beneficial to the environments as it has high efficiency of used-products reuse and low waste emissions. 
To the transfer price, the manufacturer should transfer all the unit cost savings to the retailer under the scenario of the manufacturer leads the supply chain and no one leads the supply chain. The transfer price has no impact on the steady state profit rate of the manufacturer in the presence of power retailer. When the manufacturer transfers all the unit cost savings to the retailer, the return rate is highest with no one leads the supply chain. The profitability is more determined by channel power than the product return efficiency. However, from a sustainability perspective, the channel power enhancement of the retailer would be beneficial to the environment with low waste emissions.

To develop a more general understanding of the presence of the power retailer on the closed-loop supply chain, our model could be extended to consider the case manufacturer collecting or third party collecting. Furthermore, competing retailers and manufacturers can also be worthy to be investigated, which will enhance the mathematical difficulties.

Author Contributions: Writing: J.J.M., Z.H.; Providing case and idea: Z.H.; Providing revised advice: G.L., S.-B.T., K.W., J.W.

Funding: This paper was supported in part by the National Natural Science Foundation of China with Grants Nos. 71602116, 71503165, 71571117 and 71702114, and the Innovation Ability Construction Projects for Shanghai University through Grant Nos. 15590501800, and the China Postdoctoral Science Foundation with Grant Nos. 2017M621370 and Zhongshan City Science and Technology Bureau Project (No. 2017B1015).

Conflicts of Interest: The authors declare no conflict of interest.

\section{References}

1. Savaskan, R.C.; Bhattacharya, S.; Van Wassenhove, L.N. Closed-loop supply chain models with product remanufacturing. Manag. Sci. 2004, 50, 239-252. [CrossRef]

2. Savaskan, R.C.; Van Wassenhove, L.N. Reverse channel design: The case of competing retailers. Manag. Sci. 2006, 52, 1-14. [CrossRef]

3. Mota, B.; Gomes, M.I.; Carvalho, A.; Barbosa-Povoa, A.P. Sustainable supply chains: An integrated modelling approach under uncertainty. Omega 2018, 77, 32-58. [CrossRef]

4. Ferguson, M.E.; Toktay, L.B. The effect of competition on recovery strategies. Prod. Oper. Manag. 2006, 15, 351-368. [CrossRef]

5. Govindan, K.; Soleimani, H.; Kannan, D. Reverse logistics and closed-loop supply chain: A comprehensive review to explore the future. Eur. J. Oper. Res. 2015, 240, 603-626. [CrossRef]

6. Hong, I.; Yeh, J.-S. Modeling closed-loop supply chains in the electronics industry: A retailer collection application. Transp. Res. Part E Logist. Transp. Rev. 2012, 48, 817-829. [CrossRef]

7. Huang, Z.; Nie, J. Dynamic closed-loop supply chain model with product remanufacturing. In Proceedings of the 2012 International Conference of Logistics Engineering and Management, Chengdu, China, 8-10 October 2012; Volume 2, pp. 1039-1045.

8. Wei, J.; Zhao, J. Pricing decisions with retail competition in a fuzzy closed-loop supply chain. Expert Syst. Appl. 2011, 38, 11209-11216. [CrossRef]

9. Ma, Z.; Zhang, N.; Dai, Y.; Hu, S. Managing channel profits of different cooperative models in closed-loop supply chains. Omega 2016, 59, 251-262.

10. Tsao, Y.C.; Linh, V.T.; Lu, J.C. Closed-loop supply chain network designs considering rfid adoption. Comput. Ind. Eng. 2017, 113, 716-726. [CrossRef]

11. Jena, S.K.; Sarmah, S.P.; Sarin, S. Joint-advertising for collection of returned products in a closed-loop supply chain under uncertain environment. Comput. Ind. Eng. 2017, 113, 305-322. [CrossRef]

12. Bhattacharya, R.; Kaur, A.; Amit, R.K. Price optimization of multi-stage remanufacturing in a closed loop supply chain. J. Clean. Prod. 2018, 186, 943-962. [CrossRef]

13. Kiesmüller, G.P. Optimal control of a one product recovery system with leadtimes. Int. J. Prod. Econ. 2003, 81, 333-340. [CrossRef]

14. Hosoda, T.; Disney, S.M. A unified theory of the dynamics of closed-loop supply chains. Eur. J. Oper. Res. 2018, 269, 313-326. [CrossRef]

15. Geyer, R.; Van Wassenhove, L.N.; Atasu, A. The economics of remanufacturing under limited component durability and finite product life cycles. Manag. Sci. 2007, 53, 88-100. [CrossRef] 
16. Hussain, S.S. Green consumerism and ecolabelling: A strategic behavioural model. J. Agric. Econ. 2000, 51, 77-89. [CrossRef]

17. Krikke, H.; Le Blanc, I.; van de Velde, S. Product modularity and the design of closed-loop supply chains. Calif. Manag. Rev. 2004, 46, 23-39. [CrossRef]

18. De Giovanni, P.; Zaccour, G. Cost-revenue sharing in a closed-loop supply chain. In Advances in Dynamic Games; Birkhäuser: Boston, MA, USA, 2013; Volume 12. [CrossRef]

19. Huang, Z.; Nie, J.; Tsai, S.-B. Dynamic Collection Strategy and Coordination of a Remanufacturing Closed-Loop Supply Chain under Uncertainty. Sustainability 2017, 9, 683. [CrossRef]

20. Sethi, S.; Thompson, G. Optimal Control Theory-Applications to Management Science and Economics; Springer: New York, NY, USA, 2000.

21. Liu, W.; Wei, Q.; Huang, S.Q.; Tsai, S.B. Doing Good Again? A Multilevel Institutional Perspective on Corporate Environmental Responsibility and Philanthropic Strategy. Int. J. Environ. Res. Public Health 2017, 14, 1283. [CrossRef] [PubMed]

22. Liu, W.; Shi, H.B.; Zhang, Z.; Tsai, S.B.; Zhai, Y.; Chen, Q.; Wang, J. The Development Evaluation of Economic Zones in China. Int. J. Environ. Res. Public Health 2018, 15, 56. [CrossRef] [PubMed]

23. Lin, F.H.; Tsai, S.B.; Lee, Y.C.; Hsiao, C.F.; Zhou, J.; Wang, J.; Shang, Z. Empirical research on Kano's model and customer satisfaction. PLoS ONE 2017. [CrossRef] [PubMed]

24. Peng, C.F.; Ho, L.H.; Tsai, S.B.; Hsiao, Y.C.; Zhai, Y.; Chen, Q.; Chang, L.-C.; Shang, Z. Applying the Mahalanobis-Taguchi System to Improve Tablet PC Production Processes. Sustainability 2017, 9, 1557. [CrossRef]

25. Li, H.; Zhang, H.; Tsai, S.B.; Qiu, A. China's Insurance Regulatory Reform, Corporate Governance Behavior and Insurers' Governance Effectiveness. Int. J. Environ. Res. Public Health 2017, 14, 1238. [CrossRef] [PubMed]

26. Yuan, Y.H.; Tsai, S.B.; Dai, C.Y.; Chen, H.M.; Chen, W.F.; Wu, C.H.; Li, G.; Wang, J. An Empirical Research on Relationships between Subjective Judgement, Technology Acceptance Tendency and Knowledge Transfer. PLoS ONE 2017. [CrossRef] [PubMed]

27. Chang, S.-F.; Chang, J.-C.; Lin, K.-H.; Yu, B.; Lee, Y.-C.; Tsai, S.-B.; Zhou, J.; Wu, C.; Yan, Z.-C. Measuring the service quality of e-commerce and competitive strategies. Int. J. Web Serv. Res. 2014, 11, 96-115. [CrossRef]

28. Gao, Y.; Tsai, S.-B.; Xue, X.; Ren, T.; Du, X.; Chen, Q.; Wang, J. An Empirical Study on Green Innovation Efficiency in the Green Institutional Environment. Sustainability 2018, 10, 724. [CrossRef]

29. Chou, C.-C.; Shen, C.-W.; Gao, D.; Gao, Y.; Wang, K.; Tsai, S.-B. Modelling the Dynamic Impacts of High Speed Rail Operation on Regional Public Transport-From the Perspective of Energy Economy. Energies 2018. [CrossRef]

30. Chen, Q.; Wang, J.; Yu, J.; Tsai, S.-B. An Empirical Research on Marketing Strategies of Different Risk Preference Merchant. Mat. Probl. Eng. 2018, 2018, 7947894. [CrossRef]

31. Chen, Y.; Yu, J.; Li, L.; Li, L.; Li, L.; Zhou, J.; Tsai, S.-B. An Empirical Study of the Impact of the Air Transportation Industry Energy Conservation and Emission Reduction Projects on the Local Economy in China. Int. J. Environ. Res. Public Health 2018, 15, 812. [CrossRef] [PubMed]

32. Xu, L.; Wu, Q.; Du, P.; Qiao, X.; Tsai, S.-B. Financing Target and Resale Pricing in Reward-Based Crowdfunding. Sustainability 2018, 10, 1297. [CrossRef]

33. Wu, W.; Huang, X.; Li, Y.; Chu, C.C. Optimal Quality Strategy and Matching Service on Crowdfunding Platforms. Sustainability 2018, 10, 1053. [CrossRef]

34. Su, J.; Li, C.; Tsai, S.-B.; Lu, H.; Liu, A.; Chen, Q. A sustainable closed-loop supply chain decision mechanism in the electronic sector. Sustainability 2018, 10, 1295. [CrossRef]

35. Chu, C.-C.; Tsai, S.-B.; Chen, Y.; Li, X.; Zhai, Y.; Chen, Q.; Jing, Z.; Ju, Z.-Z.; Li, B. An Empirical Study on the Relationship between Investor Protection, Government Behavior, and Financial Development. Sustainability 2017, 9, 2199. [CrossRef]

36. Nair, A.; Narasimhan, R. Dynamics of competing with quality and advertising based goodwill. Eur. J. Oper. Res. 2006, 175, 462-474. [CrossRef]

37. Huang, M.; Song, M.; Lee, L.H.; Ching, W.K. Analysis for strategy of closed-loop supply chain with dual recycling channel. Int. J. Prod. Econ. 2013, 144, 510-520. [CrossRef]

(C) 2018 by the authors. Licensee MDPI, Basel, Switzerland. This article is an open access article distributed under the terms and conditions of the Creative Commons Attribution (CC BY) license (http:/ / creativecommons.org/licenses/by/4.0/). 